\title{
Histologic Outcomes in HPV-Positive and Cervical Cytology- Negative Women - Screening Results in Northern Thailand
}

\author{
Linlada Vijakururote, Prapaporn Suprasert*, Jatupol Srisomboon, Sumalee \\ Siriaunkgul, Jongkolnee Settakorn, Sunida Rewsuwan
}

\begin{abstract}
The objective of this study was to determine the prevalence of significant lesions defined as high grade squamous intraepithelial lesions (HSIL), adenocarcinoma in situ (AIS) and invasive carcinoma in women who had HPV-positive and cytology negative co-testing screening results. This retrospective study was conducted in Chiang Mai University Hospital between May, 2013 and August, 2014. Hybrid capture 2 (HC2) was used for HPV testing and conventional Pap smears for cytologic screening. A repeat liquid-based cytology (LBC) was performed in women with such co-testing results followed by colposcopy. Random biopsy was performed in cases of normal colposcopic findings. Further investigations were carried out according to the biopsy or the repeat LBC results. During the study period, 273 women met the criteria and participated in the study. The mean age of these women was 46.4 years with $30 \%$ of them reporting more than one partner. The median interval time to colposcopy was 165 days. About $40 \%$ showed an abnormality in the repeat cytology. Significant cervical lesions were found in $20(7.3 \%)$ women, including 2 invasive cancers. Of interest was that only 2 of 20 significant lesions were diagnosed by colposcopic examination while the remainder were initially detected by cervical biopsy and abnormal repeat cytology. In conclusion, the prevalence of significant cervical lesions in HPV positive and cytology negative women in Northern Thailand was $\mathbf{7 . 3 \%}$. Further diagnostic work up with repeat cytology follow by colposcopy is recommended. Random biopsy should be performed even when the colposcopic findings are normal.
\end{abstract}

Keywords: Positive HPV test - negative cytology - liquid-based cytology - random biopsy

Asian Pac J Cancer Prev, 16 (16), 7271-7275

\section{Introduction}

Cervical cancer is currently recognized as the most preventable cancer via primary prevention with HPV vaccination and secondary prevention with regular screening utilizing various methods. Testing methods include cervical cytology with either a conventional Pap smear or liquid-based cytology, HPV testing with or without cytology and visual inspection with acetic acid. The aim of cervical cancer screening is early detection and prompt treatment of the precancerous lesions including cervical intraepithelial neoplasia (CIN) grade 2, CIN 3 commonly known as high-grade squamous intraepithelial lesions (HSIL) and adenocarcinoma in situ (AIS).

At present, it is well established that persistent cervical HPV infection is preliminary to the development of cervical cancer and other precancerous lesions. Nearly $100 \%$ of HPV is detected in cervical cancer (Bosch et al., 2002; Siriaunkgul et al., 2008; Sanjose et al., 2010). According to the 2012 American Cancer Society, American Society for Colposcopy and Cervical Pathology, and the American Society for Clinical Pathology (ASCCP) screening guidelines, the preferred screening method for women aged 30-65 years was a combination of HPV testing and cervical cytology known as co-testing. Women with HPV-negative and cytology-negative co-testing can be re-screened every five years, while women with HPV-positive and cytology-negative results are at risk for not only having underlying HSIL but also developing such lesions in the future (Saslow et al., 2012). The recommended guidelines for managing HPV-positive but cytology-negative women is to either repeat co-testing at one year or perform HPV 16/18 genotyping. If the co-test results at one year is a positive HPV test or the cytology has atypical squamous cells of undetermined significance (ASC-US) or worse, colposcopy is recommended. If HPV16 or HPV18 tests were positive, a colposcopy was also recommended (Saslow et al., 2012). However, previous studies in the Western countries where the incidence of cervical cancer was low, have reported that the risk of having underlying HSIL in women with HPVpositive and cytology-negative results was approximately 
Linlada Vijakururote et al

3-7 \% (Thrall et al., 2010; Wright et al., 2011). In Thailand, 2 publications from the north and northeastern regions of Thailand reported a different prevalence of HSIL in HPVpositive and cytology negative women at $11 \%$ and $2.4 \%$, respectively. Both studies utilized different methods for HPV testing (Paengchit et al., 2014; Supho et al., 2014). Due to the variation of prevalence of significant lesions in Thailand, we conducted a retrospective study to evaluate the prevalence of significant lesions in women who tested HPV positive only from co-testing cervical cancer screening at our institute. More knowledge regarding prevalence would be beneficial in counseling and planning appropriate management for the Thai women having such screening results.

\section{Materials and Methods}

After approval by the Research Ethics Committee of the Faculty of Medicine, Chiang Mai University, women at least 30 years of age with HPV-positive and cytologynegative from a cervical cancer co-test screening program that underwent colposcopy examination at Chiang Mai University Hospital between May 1, 2013 and August 1, 2014 were reviewed. Pregnant women or women with a prior abnormal cytology or positive HPV testing or previous treatment for cervical neoplasia were excluded.

All studied women participated in the cervical cancer screening project from three districts in Chiang Mai Province (Sankampang, Mae-on and Sarapee). They received co-test screening using conventional Pap smear and HPV testing. The cervical specimen was collected by "digene cervical sampler and collecting tube" for HPV testing (HC2). The Pap smears were screened by cytotechnologists and sign-out if they were "negative for epithelial abnormality. Abnormal or questionable cases were referred to cytopathologists. Cytologic interpretation by both cytotechnologists and cytopathologists were blinded to the HPV testing results. The HPV test was the Hybrid Capture 2 (HC2; Qiagen, Hilden, Germany that used the signal amplification technique to test for the pool of high-risk HPVs according to the manufacturers' instruction. The signal strength was proportional to the HPV load in the sample. The clinically validated cut-off value of the HC2 was $1 \mathrm{pg} / \mathrm{ml}$ or approximately 5,000 copies per sample. The HC2 detects the presence of 13 high-risk HPV genotypes including HPVs 16, 18, 31, 33, $35,39,45,51,52,56,58,59$ and 68. Positive HPV test of the $\mathrm{HC} 2$ was defined with a quantitative threshold of a relative light unit/positive control (RLU/PC) ratio $>1$.

Colposcopy was carried out by gynecologic oncologists. At the starting process of a colposcopy, the liquid-based cytology (LBC) was repeated. After colposcopic examination and the impression was concluded, a colposcopically directed biopsy (CDB) was performed at the most abnormal appearance area. In cases of normal colposcopic findings or an unsatisfactory finding, cervical biopsy was performed at 6 and 12 o'clock of the cervix at or near the transformation zone. A further investigation procedure to achieve the final histology depended on the results of the CDB and the results of the repeat cytology. The final histologic diagnosis was made on the most severe histologic results obtained from either $\mathrm{CDB}$, loop electrosurgical excision procedure (LEEP) or hysterectomy and was categorized for analysis as normal/ inflammation, low-grade squamous intraepithelial lesions (LSIL), HSIL and/or invasive carcinoma. Significant cervical lesions were defined as HSIL, AIS, and invasive carcinoma. All specimens obtained were interpreted by experienced gynecologic pathologists. The patient demographics, the clinical data and the histologic results were recorded.

Statistical analysis of the data was carried out using the SPSS for Window program (Version 17.0, Chicago, IL, USA). Descriptive data of all studied patients were presented as means with range and discrete data were reported as number and percentages.

\section{Results}

Two hundred and seventy three medical records were eligible and reviewed. The clinical characteristics were presented in Table 1. The mean age of these women was 46.39 years with about one-third of them in the climacteric period and only one case reported using hormonal replacement therapy. About $90 \%$ were multiparous and the most frequent contraceptive used was tubal resection while $45 \%$ reported no contraception usage. The mean age of initial sexual intercourse was 20 years and almost half of the studied patients reported more than one partner. Only $2.9 \%$ of the studied women were current smokers and $5.1 \%$ were HIV positive. The median time between cervical cancer screening and the colposcopic procedure was 165.0 days.

Regarding the LBC taken before colposcopic examination in 273 women, $176(64.5 \%)$ were negative for epithelial abnormality. Atypical squamous cell of undetermined significant (ACS-US) cytology was the most common cytology abnormality accounting for 44 cases $(16.1 \%)$ followed by LSIL cytology (35 cases). The remaining were HSIL cytology ( 8 cases), atypical squamous cell could not be exclude HSIL or ASC-H ( 8 cases) and unsatisfied smear (1 case). The repeat LBC was not performed in 1 patient.

Among 273 women, 20 (7.32\%) had significant lesions from the final histology. The relationship between colposcopic impression and the final histology was shown in Table 2 . About $75 \%$ of the studied patients had satisfactory colposcopic findings. Of interest, only one case of HSIL and one case of AIS could be diagnosed by colposcopic findings while almost all of the significant lesions were perceived as normal and LSIL by colposcopy.

The details of all 20 patients who revealed significant lesions were summarized in Table 3 . The median age of these patients was 47.0 years and 5 were menopausal women. Most of them were multiparous and about $20 \%$ of the patients were anti-HIV positive. Nine women reported that the initial sexual intercourse occurred in the adolescent period and six cases had more than one sexual partner. All except one patient showed abnormal repeat LBC with 12 presenting with HSIL (7 cases) and ASC-H (5 cases). The most frequent initial detection method for a significant lesion was a cervical biopsy 
during colposcopy that found HSIL in 12 patients, AIS in 2 patients and cancer in 1 patient. Three patients were detected later by subsequent LEEP that was performed due to cytologic results from LBC before colposcopy showing HSIL in 2 cases and ASC-H in 1 case. The final histology was HSIL in 16 cases, AIS in 2 cases and squamous cell carcinoma in the 2 patients. One cancer case was 39 years old and was diagnosed as stage IIB with an infiltrative cervical lesion of $2 \mathrm{~cm}$. with left parametrial metastasis. The colposcopic finding was unsatisfactory and random biopsy showed squamous cell carcinoma while the repeat cytology showed ASC-H. This patient was treated with concurrent chemoradiation. The remaining one patient with invasive cancer was 46 years old. The impression from colposcopy was LSIL but the CDB showed HSIL that corresponded to the repeat LBC. She underwent LEEP and the histology revealed squamous cell carcinoma, moderate differentiation with a depth of invasion of 2.5 $\mathrm{mm}$. and width of invasion $6 \mathrm{~mm}$. without lymphovascular space invasion. She was diagnosed as stage IA1 cervical cancer and was referred to undergo a simple hysterectomy at the local hospital. One patient had AIS lesions in both the cervical biopsy at colposcopy and subsequent LEEP specimens without abnormal results of the repeat $\mathrm{LBC}$, while another patient had AIS in the cervical biopsy specimen but had HSIL in LEEP specimen and the repeat LBC showed only ASC-US.

Table 1. Clinical Characteristics $(\mathrm{N}=\mathbf{2 7 3})$

\begin{tabular}{lr}
\hline & $\mathrm{N}(\%)$ \\
\hline Mean Age (SD) & $46.39(8.44)$ \\
Menopause & $94(34.4)$ \\
Nulliparous & $29(10.6)$ \\
HIV Positive & $14(5.1)$ \\
Contraception & \\
$\quad$ Tubal Resection & $62(22.7)$ \\
Oral Contraceptive Pill & $39(14.3)$ \\
DMPA & $30(11.0)$ \\
Condom & $12(4.4)$ \\
Intrauterine Device & $5(1.8)$ \\
None & $125(45.8)$ \\
Median Age of First Sexual Intercourse (Year;Range) & $20.00(13-40)$ \\
Number of Partner(s) & \\
1 & $158(57.9)$ \\
2 & $82(30.0)$ \\
3 & $27(9.98)$ \\
4 & $3(1.1)$ \\
5 & $1(0.4)$ \\
6 & $2(0.7)$ \\
Current Smoker & $8(2.9)$ \\
Median Interval Time to Colposcopy (Range:Days) & $165.0(12-291)$
\end{tabular}

Table 2. The Relationship of Final Pathology to Colposcopic Impression

\begin{tabular}{lcccccc}
\hline Final & Normal & LSIL & HSIL & AIS & Cancer & Total \\
\hline Impression & & & & & & \\
$\quad$ Normal & 52 & 14 & 6 & 0 & 0 & 72 \\
LSIL & 79 & 33 & 7 & 1 & 1 & 121 \\
HSIL & 2 & 3 & 1 & 1 & 0 & 7 \\
Unsatisfactory & 61 & 9 & 2 & 0 & 1 & 73 \\
\hline Total & 194 & 59 & 16 & 2 & 2 & 273 \\
\hline
\end{tabular}

LSIL = low grade squamous intraepithelial lesion; HSIL = high grade squamous intraepithelial lesion; AIS = adenocarcinoma in situ
Table 3. Characteristics of Patients with Final Histology of Significant Lesions ( $N=20)$

$\mathrm{N}(\%)$

Median Age (Range)

Menopause

$47.00(24-58)$ years

Parity

0

$5(25.0)$

1

2

$3(15.0)$

HIV Positive

$10(50.0)$

$7(35.0)$

Contraception

$4(20.0)$

None

$6(30.0)$

Tubal Resection

$6(30.0)$

Oral Contraceptive Pill 3(15.0)

DMPA

$3(15.0)$

Condom

$3(15.0)$

First Sexual Intercourse (Years)

15-20

$9(45.0)$

$21-25$

$8(40.0)$

26-30

$3(15.0)$

Number of Partner(s)

1

$14(70.0)$

$5(25.0)$

$1(5.0)$

3

$1(5.0)$

Smoker

$57.5(39-215)$

Repeated Cytology Result

Negative $1(5.0)$

LSIL $1(5.0)$

HSIL 7(35.0)

ASC-US 6(30.0)

ASC-H 5(25.0)

The Initial Detection Method

CDB

$17(85.0)$

LEEP

$3(15.0)$

Final Histology

HSIL

$16(80.0)$

AIS

2(10.0)

SCCA

$\mathrm{CDB}=$ colposcopically directed biopsy; LEEP = loop electrosurgical excision procedure; HSIL = high - grade
squamous intraepithelial lesions; AIS = adenocarcinoma in situ; SCCA = squamous cell carcinoma

\section{Discussion}

The prevalence of significant lesions in women who had HPV-positive and cytology-negative screening results in the present study was $7.3 \%$ which was concordant with previous studies (Thrall et al., 2010; Wright et al., 2011). However, when compared to the other studies in Thailand, the prevalence was a little bit lower from that of $11 \%$ in Lampang province locating in northern Thailand (Paengchit et al., 2014) and was much higher than that of $2.4 \%$ in Khon Kaen province locating in the northeastern region of Thailand (Supho et al., 2014). The Northeastern study (Supho et al., 2014) did not mention the type of HPV test and did not perform a random biopsy in patients who had negative colposcopy findings unlike the northern study and the present study. The northern study (Paengchit et al., 2014) which used an EIA kit HPV GP HR (Diassay Rijswijk, The Netherlands) for HPV testing and carried out random biopsy in women with normal colposcopic findings. The random biopsy performed in 
women who had a negative finding from colposcopy in the northern study and the present study seemed to increase the detection of significant lesions. Among 20 patients with significant lesions in the present study, 4 patients who had normal colposcopic finding were detected such significant lesions by random biopsy. This finding was supported by the previous study (Song et al., 2015) noting that random biopsy was beneficial in women with positive HPV testing who had normal colposcopic findings.

The failure to detect HSIL from colposcopy in the present study corresponded to a recent study from India (Ghosh et al., 2014). The Indian noted a very poor agreement with kappa only 0.17 between the colposcopic finding and the final histology of colposcopy in an HPV detection-based cervical cancer program of over 2000 women. In addition, over $50 \%$ of HSIL in their study could not be detected by colposcopic examination. They explained that such low accuracy of colposcopic impression might be from the hypothesis of thin epithelial lesion of CIN as mentioned by Yang et al (2008). Increased accuracy for the diagnosis of HSIL seemed to be from random biopsy as mentioned above. Furthermore, the repeat LBC before colposcopy was beneficial to increase the detection rate of significant lesions. As in the present study, about $40 \%$ of the repeat LBC that performed at the median interval time of 5 months showed abnormality. From this data, the recommendation of ASCCP guidelines (Saslow et al., 2012) that suggested co-testing at one year in such women might not be appropriate for Thai northern women. Instead, we would like to recommend the optimal time to repeat the cytology at 6 months.

The type of HPV testing, HC2 that was used in the present study showed similar sensitivity when compared with the Cobas 4800 and the Abbott RT HPV tests (Arbyn et al., 2012). The inferiority of HC2 test was the inability to identify the genotype of HPV as in the Cobas 4800 or identified mRNA of 14 HR-HPV in the APTIMA test. As a result, these 2 tests had higher specificity than the HC2 test for detection of HSIL (Arbyn et al., 2012). Many previous publications revealed higher prevalence of significant lesions in women with positive HPV type 16,18 and HPV mRNA (Wright et al., 2011; Arbyn et al., 2012; Wright et al., 2012; Paengchit et al., 2014).

Neither of the 2 women in whom AIS was detected showed abnormal cytology in the screening and repeat settings. It is well recognized that cytological testing is less effective in detecting invasive adenocarcinoma and its precursor, AIS. Lesions of the AIS preferentially extend into the endocervical canal and occur within the endocervical clefts rather than at the surface of transformation zone as with the squamous lesions. Consequently, cytological screening frequently fails to detect such lesions. In addition, glandular lesions have no specific colposcopic features that help differentiate it from the squamous lesions. In our previous study, combined Pap smear and colposcopy had a sensitivity of only $61 \%$ in detecting AIS lesions, supporting what is known about the limitations of conventional methods in identifying AIS lesions (Kietpeerakool et al., 2006). In the clinical trials of quadrivalent HPV vaccine, among 22 women with AIS, only two had glandular abnormalities on cervical cytology, furthermore, colposcopy failed to detect all AIS lesions (Ault et al., 2011). These findings provided strong support for incorporating HPV testing into cytological screening to better identify women at risk of having AIS. The higher sensitivity of HPV testing combined with cervical cytology helped increase the effectiveness of screening for adenocarcinoma (Katki et al., 2011; Andersson et al., 2013).

The limitations of this study were the inability to evaluate the influence of HPV genotype 16/18 on the risk of underlying significant lesions in women who had positive HPV test and negative cytology and only the current or baseline risk of significant lesions was analyzed, the impact of HPV persistence and risk factors for such lesions were not assessed due to the small number of patients. However, in the present study, all pathology specimens were interpreted by experienced gynecologic pathologists and all histologic data were completely recorded which made the results of this study reliable. In conclusion, the prevalence of significant lesions in women who had positive - HPV testing only was $7.3 \%$. Further investigations with immediate colposcopy is recommended. Random cervical biopsies should be performed even though the colposcopic appearance is normal.

\section{Acknowledgements}

We wish to thank the National Research University Project under Thailand's Office of Higher Education Commission and Chiang Mai University for the financial support in this project.

\section{References}

Andersson S, Mints M, Wilander E (2013). Results of cytology and high-risk human papillomavirus testing in females with cervical adenocarcinoma. Oncol Lett, 6, 215-9.

Arbyn M, Ronco G, Anttila A, et al (2012). Evidence regarding human papillomavirus testing in secondary prevention of cervical cancer. Vaccine, 30, 88-99.

Ault KA, Joura EA, Kjaer SK, et al (2011). Adenocarcinoma in situ and associated human papillomavirus type distribution observed in two clinical trials of a quadrivalent human papillomavirus vaccine. Int J Cancer, 128, 1344-53.

Bosch FX, Lorincz A, Muñoz N, et al (2001). The causal relation between human papillomavirus and cervical cancer. J Clin Pathol, 55, 244-65.

Ghosh I, Mittal S, Banerjee D, et al (2014). Study of accuracy of colposcopy in VIA and HPV detection-based cervical cancer screening program. Aust N Z J Obstet Gynaecol, 54, 570-5.

Katki HA, Kinney WK, Fetterman B, et al (2011). Cervical cancer risk for women undergoing concurrent testing for human papillomavirus and cervical cytology: a populationbased study in routine clinical practice. Lancet Oncol, 12, 663-72.

Kietpeerakool C, Srisomboon J, Prompittayarat W, et al (2006). Can adenocarcinoma in situ of the uterine cervix be predicted before cervical conization? Asian Pac J Cancer Prev, 7, $522-4$.

Paengchit K, Kietpeerakool C, Wangchai W, et al (2014). Cervical pathology in cytology-negative/HPV-positive women: results from Lampang Cancer Hospital, Thailand. 

Asian Pac J Cancer Prev, 15, 7951-54.

Song Y, Zhao YQ, Zhang X, et al (2015). Random biopsy in colposcopy-negative quadrant is not effective in women with positive colposcopy in practice. Cancer Epidemiol, 39, $237-41$.

Supho B, Supoken A, Kleebkaew P, et al (2014). Cervical pathology in high-risk human papillomavirus- positive, cytologically normal women. Asian Pac J Cancer Prev, 15, 7977-80.

Saslow D, Solomon D, Lawson HW, et al (2012). American Cancer Society, American Society for Colposcopy and Cervical Pathology, and American Society for Clinical Pathology screening guidelines for the prevention and early detection of cervical cancer. CA Cancer J Clin, 62, 147-72.

de Sanjose S, Quint WG, Alemany L, et al (2010). Human papillomavirus genotype attribution in invasive cervical cancer: a retrospective cross-sectional worldwide study. Lancet Oncol, 11, 1048-56.

Siriaunkgul S, Suwiwat S, Settakorn J, et al (2008). HPV genotyping in cervical cancer in Northern Thailand: adapting the linear array HPV assay for use on paraffin-embedded tissue. Gynecol Oncol, 108, 555-60.

Thrall MJ, Russell DK, Facik MS, et al (2010). High-risk HPV testing in women 30 years or older with negative Papanicolaou tests: initial clinical experience with 18-month follow-up. Am J Clin Pathol, 133, 894-8.

Wright TC Jr, Stoler MH, Behrens CM, et al (2012). The ATHENA human papillomavirus study: design, methods, and baseline results. Am J Obstet Gynecol, 206, 1-46.

Wright TC Jr, Stoler MH, Sharma A, et al (2011). Evaluation of HPV-16 and HPV-18 genotyping for the triage of women with high-risk HPV+ cytology-negative results. Am J Clin Pathol, 136, 578-86.

Yang B, Pretorius RG, Belinson JL, et al (2008). False negative colposcopy is associated with thinner cervical intraepithelial neoplasia 2 and 3. Gynecol Oncol, 110, 32-6 